\begin{tabular}{|c|l|}
\hline Title & A symplectic framework for multiplane gravitational lensing \\
\hline Author(s) & Izumiya, S.; Janeczko, S. \\
\hline Citation & JOURNAL OF MA THEMATICAL PHY SICS, 44(5), 2077-2093 \\
\hline https:/doi.org/40.1063/1563042 \\
\hline Issue Date & 2003-05 \\
\hline Doc URL & http://hdl.handle.net/2115/5910 \\
\hline Rights & Copyright $\odot 2003$ A merican Institute of Physics \\
\hline Type & article \\
\hline File Information & JMP44.5.pdf \\
\hline
\end{tabular}

Instructions for use 


\title{
A symplectic framework for multiplane gravitational lensing
}

\author{
S. Izumiya ${ }^{\text {a) }}$ \\ Department of Mathematics, Hokkaido University, Sapporo 060-0810, Japan \\ S. Janeczko \\ Institute of Mathematics, PAN, ul. Sniadeckich 8, 00-950 Warsaw, Poland \\ and Faculty of Mathematics and Inf. Sci., Warsaw University of Technology, \\ Pl. Politechniki 1, 00661 Warsaw, Poland
}

(Received 16 October 2002; accepted 24 January 2003)

\begin{abstract}
We construct a new framework for the study of multiplane gravitational lensing from the view point of symplectic geometry. Symplectic relations are used to compose the systems and weaker Lagrangian equivalence is applied for classifying the caustics of multiplane gravitational lensing. (C) 2003 American Institute of Physics.
\end{abstract}

[DOI: 10.1063/1.1563042]

\section{INTRODUCTION}

Recently there appeared several articles considering gravitational lensing systems as applications of the theory of singularities for smooth mappings. The gravitational lensing is the deflection of light from a distant source (e.g., quasar) by an intervening matter distribution (e.g., a galaxy or a cluster of galaxies). The first gravitational lensed quasar was detected only in 1979. By now gravitational lensing is quite an active field in astrophysics. ${ }^{1-3}$

On the other hand, singularity theory of Lagrangian varieties ${ }^{4,5}$ is the best natural setting for discussing optical systems. In fact, Petters and his collaborators ${ }^{6,1}$ pointed out that a single gravitational lensing can be described in the framework of symplectic geometry. Especially the caustics in a single gravitational lensing system coincide with caustics in the theory of Lagrangian singularities. Moreover, they also investigate multiplane gravitational lensing as an application of singularity theory. ${ }^{7,1}$ The standard treatment of gravitational lensing uses a notion of equivalence that yields either folds or cusps as the locally stable caustics for a $k$-plane lensing map (e.g., in Ref. 1). On the other hand, Levine and Petters ${ }^{8}$ speculated that under a weaker notion of equivalence, some caustics other than folds and cusps would appear stable for lens systems exposed to a more restricted family of perturbations. However, in their framework for multiplane gravitation lensing generic caustics are the same as those for the single gravitational lensing. Current observed lensed systems fit with the standard notion of equivalence used in the lensing literature (where only folds or cusps appear). However, as instruments discover more and more lens systems, it may be possible to find a system that is exposed to more limited family of perturbations and for which caustics like handkerchief, etc., appear stable possibly for a "short" time period. Note that on cosmic time scales (i.e., of the order billions of years) events that last months or a few years are quite short, though such time periods are long enough on human time scales for us to carry out observations.

In this paper we propose the symplectic framework for multiplane gravitational lensing based on the notion of symplectic relation, which is a natural generalization of the notion of symplectic transformation (cf. Ref. 9). The original motivation for the paper was an attempt to describe expected nonstandard caustics in gravitational lensing using the weaker versions of Lagrangian equivalence acting on composite symplectic relations. These composites correspond to images of

${ }^{a)}$ Electronic mail: izumiya@math.sci.hokudai.ac.jp 
systems of gravitational rays by the subsequent gravitational lens, and their generic forms, described in the paper, reflect the complexity of composition.

In Sec. II we introduce the gravitational lensing problem and in Sec. III the problem is formulated in the language of Lagrangian stability and versality adapted to the product symplectic space of incoming and outgoing rays. The precise meaning of the composition of systems and their actions on the subsequent wave fronts represented by generating functions was introduced in Sec. IV. In Sec. V the local stability of double lensing systems was investigated and it is continued in Sec. VI by classification of the normal forms of generating pairs with respect to the natural groups of equivalences.

All manifolds and maps considered here are of class $C^{\infty}$ unless stated otherwise.

\section{GRAVITATIONAL LENSING}

In this section we give a quick review of the basic concepts from the theory of gravitational lensing discussed already in Refs. 8, 7, 6, 10, 1, and 3.

(1) Single lensing ( $c f .$, Refs. 6, 1, and 3). Consider the typical single lens plane gravitational lensing as follows: We assume that the deflector is thin and apply the small angle approximation (cf., Ref. 6). The extra time with respect to the unperturbed ray is giving the time-delay map; $T_{\mathrm{s}}: \mathbb{R}^{2} \supset U \rightarrow \mathbb{R}$ defined by

$$
T_{\mathbf{s}}(\mathbf{r})=\left(1+z_{L}\right)\left[\frac{d_{O L} d_{O S}}{2 d_{L S}}\left\|\frac{\mathbf{s}}{d_{O S}}-\frac{\mathbf{r}}{d_{O L}}\right\|^{2}-\Psi(\mathbf{r})\right] .
$$

Here, $z_{L}$ is the redshift of the lens plane, $d_{O L}, d_{O S}, d_{L S}$ are angular diameter distances, $\mathbf{r}$ is the position on the lens plane where the ray hits, $\mathbf{s}$ is the position of the source, and $\Psi(\mathbf{r})$ is the two-dimensional potential of the deflector on the lens plane. The deflector potentials $\Psi$ occurring in the time-delay map are given by

$$
\Psi(\mathbf{r})=4 \int_{\mathbb{R}^{n}} \sigma\left(\mathbf{r}^{\prime}\right) \ln \left\|\frac{\mathbf{r}^{\prime}-\mathbf{r}}{d_{O L}}\right\| .
$$

They are solutions of two-dimensional Poisson equation $\Delta \Psi(\mathbf{r})=8 \pi \sigma(\mathbf{r})$, where $\sigma(\mathbf{r})$ is the surface mass density (cf., Fig. 1).

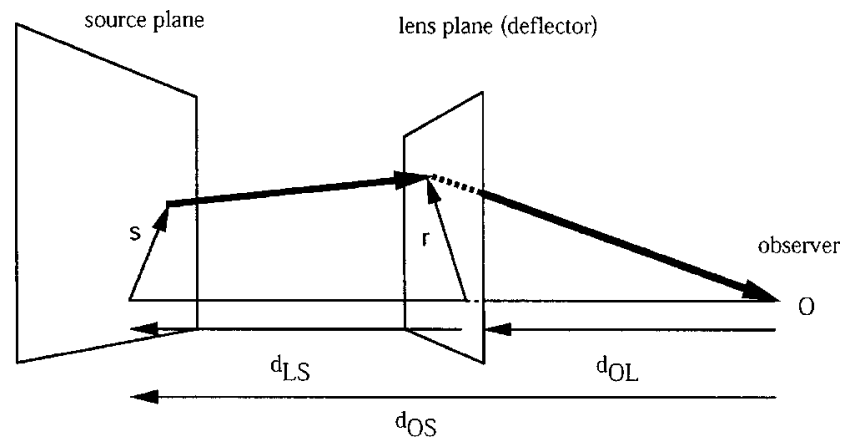

FIG. 1. A single lensing diagram. Angles are exaggerated. The distances are significantly larger than the diameter of the lens. 
By suitable coordinate transformations, we can express the time-delay map in the convenient form:

$$
T_{\mathbf{y}}(\mathbf{x})=\Theta_{L}\left[\frac{\|\mathbf{x}-\mathbf{y}\|^{2}}{2}-\psi(\mathbf{x})\right] \quad\left(\mathbf{x}, \mathbf{y} \in \mathbb{R}^{2}\right) .
$$

Here $\mathbf{y}$ corresponds to the point on the source $\mathbf{s}$ plane and $\mathbf{x}$ corresponds to the point on the lens plane $\mathbf{r}$.

Fermat's principle yields the critical points of the time delay map $T_{\mathbf{y}}(\mathbf{x})$ with respect to variations in $\mathbf{x}$ determining those rays that are real light rays (cf., Ref. 6). For this reason, a critical point of $T_{\mathbf{y}}(\mathbf{x})$ relative to $\mathbf{x}$ is called an image of the point source at $\mathbf{y}$. The magnification of an image $\mathbf{x}$ of a source at $\mathbf{y}$ is defined by

$$
A_{\mathbf{y}}(\mathbf{x})=\frac{1}{\left\|\operatorname{det} T_{\mathbf{x x}}(\mathbf{x} ; \mathbf{y})\right\|},
$$

where $T(\mathbf{x} ; \mathbf{y})=T_{\mathbf{y}}(\mathbf{x})$ and $T_{\mathbf{x x}}(\mathbf{x} ; \mathbf{y})$ is the Hessian matrix with respect to $\mathbf{x}$. A caustic point in gravitational lensing is a position $\mathbf{y} \in \mathbb{R}^{2}$ for which a source at $\mathbf{y}$ will have at least one image of infinite magnification. In other words, caustics are source positions $\mathbf{y} \in \mathbb{R}^{2}$ for which the timedelay map $T_{\mathbf{y}}(\mathbf{x})$ has at least one degenerate critical point (i.e., $\operatorname{det} T_{\mathbf{x x}}(\mathbf{x} ; \mathbf{y})=0$ ). So, we may consider that the time-delay map is the generating family of a certain Lagrangian submanifold in $T^{*} \mathbb{R}^{2}$ (cf., Sec. III).

(2) Multiplane gravitational lensing (cf., Refs. 8, 10, 1, and 3). Although we can consider a general $k$-planes gravitational lensing, we now only consider the case when $k=2$ (i.e, a double plane gravitational lensing) for convenience.

The typical double lens plane gravitational lensing situation is given as follows: There are two lens planes with "thin" deflectors in each plane. The deflectors are assumed to be independent, that is, the lens planes are sufficiently spaced so that they do not interact. Furthermore, the small angle approximation is assumed. We also parametrize all rays originating from the point source at $\mathbf{s}$, deflected by two gravitational lens, using the four-dimensional vectors $\left(\mathbf{r}_{1}, \mathbf{r}_{2}\right)$. Relative to these approximations the extra time $T_{\mathrm{s}}$ to reach the indicated observer from $\mathbf{s}$ is given by the time-delay map. It is the function $T_{\mathrm{s}}: U_{1} \times U_{2} \subset \mathbb{R}^{4} \rightarrow \mathbb{R}$ with each domain $U_{i} \subset \mathbb{R}^{2}$ being an open subset, defined by

$$
T_{\mathbf{s}}\left(\mathbf{r}_{1}, \mathbf{r}_{2}\right)=\sum_{i=1}^{2}\left(1+z_{i}\right)\left[\frac{d_{i} d_{i+1}}{2 d_{i, i+1}}\left\|\frac{\mathbf{r}_{i+1}}{d_{i+1}}-\frac{\mathbf{r}_{i}}{d_{i}}\right\|^{2}-\Psi_{i}\left(\mathbf{r}_{i}\right)\right] .
$$

Here, $z_{i}$ is the redshift of the $i$ th lens plane, $d_{i j}$ is the angular diameter distance separating the $i$ th and $j$ th lens planes, $d_{i}$ is the angular diameter distance from the observer to the $i$ th lens plane with $d_{k+1} \equiv d_{S}$ the distance to the source plane, $\mathbf{r}_{i}$ is the position on the $i$ th plane where the ray hits, $\mathbf{r}_{k+1} \equiv \mathbf{s}$, and $\Psi_{i}\left(\mathbf{r}_{i}\right)$ is the two-dimensional potential of the deflector on the $i$ th lens plane (cf., Fig. 2).

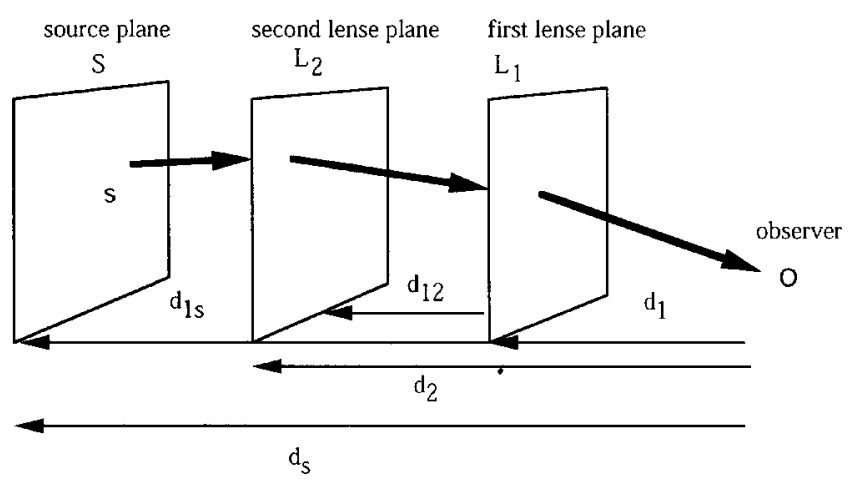

FIG. 2. A ray diagram for double plane gravitational lensing. 
By suitable coordinate transformations, the double plane time-delay map can be expressed conveniently as follows:

$$
T_{\mathbf{y}}\left(\mathbf{x}_{1}, \mathbf{x}_{2}\right)=\Theta_{1}\left[\frac{\left\|\mathbf{x}_{2}-\mathbf{x}_{1}\right\|^{2}}{2}-\beta_{1,2} \psi_{1}\left(\mathbf{x}_{1}\right)\right]+\Theta_{2}\left[\frac{\left\|\mathbf{x}_{2}-\mathbf{y}\right\|^{2}}{2}-\beta_{2,3} \psi_{2}\left(\mathbf{x}_{2}\right)\right], \quad\left(\mathbf{x}_{1}, \mathbf{x}_{2}, \mathbf{y} \in \mathbb{R}^{2}\right) .
$$

In Ref. 10, Fermat's principle has been adapted exactly in the same way as it was used already for the single lens plane case, so that the image of a gravitational lensed point like light source at position $\mathbf{y}$ are identified to the critical points of $T_{\mathbf{y}}$, e.g., the set of images is given as follows:

$$
\left\{\left(\mathbf{x}_{1}, \mathbf{x}_{2}\right) \mid \operatorname{grad}_{\mathbf{x}_{i}} T_{\mathbf{y}}\left(\mathbf{x}_{1}, \mathbf{x}_{2}\right)=\mathbf{0}, \quad i=1,2\right\} .
$$

If we adapt this principle, then the classification of caustics for single and multiple lens planes is the same, namely, folds and cusps. It is, however, pointed out in Ref. 8 that double folds or handkerchiefs might appear as the stable caustics for double plane lensing under a more restricted family of perturbations. These singularities do not appear as generic caustics under the abovementioned construction. Therefore, our opinion is that we need to have another interpretation of Fermat's principle.

\section{A SYMPLECTIC FRAMEWORK FOR SINGLE GRAVITATIONAL LENSING}

In Ref. 6 Petters pointed out that single gravitational lensing can be described in the framework of symplectic geometry (i.e, Lagrangian singularity theory). In the first place we briefly review the Lagrangian singularity theory. ${ }^{4}$ Let $\pi: T^{*} \mathbb{R}^{n} \rightarrow \mathbb{R}^{n}$ be the cotangent bundle over $\mathbb{R}^{n}$. We may consider that $T^{*} \mathbb{R}^{n}=\mathbb{R}^{2 n}$ and $\pi\left(q_{1}, \ldots, q_{n} ; p_{1}, \ldots, p_{n}\right)=\left(q_{1}, \ldots, q_{n}\right)$, where $\left(q_{1}, \ldots, q_{n} ; p_{1}, \ldots, p_{n}\right)$ are the canonical coordinates on $T^{*} \mathbb{R}^{n}$. There exits the Liouville oneform $\alpha=\sum_{i=1}^{n} p_{i} d q_{i}$ on $T^{*} \mathbb{R}^{n}$. We call the two-form $\omega=d \alpha=\sum_{i=1}^{n} d p_{i} \wedge d q_{i}$ the canonical symplectic structure on $T^{*} \mathbb{R}^{n}$. A Lagrangian submanifold $\iota: L \subset T^{*} \mathbb{R}^{n}$ is a submanifold with $L=n$ and $\iota^{*} \omega=0$. We call a map $\pi \circ \iota: L \rightarrow \mathbb{R}^{n}$ a Lagrangian map.

There is the notion of generating families for Lagrangian immersion germs as follows: Define an $n$-parameter family of function germs $F:\left(\mathbb{R}^{k} \times \mathbb{R}^{n}, 0\right) \rightarrow(\mathbb{R}, 0)$ to be a Morse family if the map germ

$$
\frac{\partial F}{\partial \lambda}:\left(\mathbb{R}^{k} \times \mathbb{R}^{n}, 0\right) \rightarrow\left(\mathbb{R}^{k}, 0\right)
$$

is nonsingular, where $\partial F / \partial \lambda(\lambda, q)=\left(\partial F / \partial \lambda_{1}(\lambda, q), \ldots, \partial F / \partial \lambda_{k}(\lambda, q)\right)$. It follows that $\Sigma(F)$ $=(\partial F / \partial \lambda)^{-1}(0)$ is a smooth submanifold germ in $\left(\mathbb{R}^{k} \times \mathbb{R}^{n}, 0\right)$. For a Morse family $F$, we define a map germ

$$
\Phi_{F}: \Sigma(F) \rightarrow T^{*} \mathbb{R}^{n}, \quad F_{F}(\lambda, q)=\left(q, \frac{\partial F}{\partial q}(\lambda, q)\right) .
$$

Then it is easy to see that $\Phi_{F}$ is a Lagrangian immersion germ. We also have the following well-known result: ${ }^{4}$

Proposition 3.1: All Lagrangian immersion germs are constructed by the above-presented method.

We call $F$ a generating family of the Lagrangian submanifold germ $\Phi_{F}(\Sigma(F))$. By Proposition 3.1, we can interpret the local property of Lagrangian immersions by using the notion of generating family, so that the singularity theory of function germs has been applied. ${ }^{4}$

There is a natural equivalence among Lagrangian map germs. Let $\pi^{\circ} \iota_{i}:\left(L_{i}, z_{i}\right)$ $\rightarrow\left(R^{n}, \pi\left(z_{i}\right)\right)(i=1,2)$ be Lagrangian map germs. We say that $\pi^{\circ} \iota_{1}:\left(L_{1}, z_{1}\right) \rightarrow\left(\mathbb{R}^{n}, \pi\left(z_{1}\right)\right)$ and $\pi \circ \iota_{2}:\left(L_{2}, z_{2}\right) \rightarrow\left(\mathbb{R}^{n}, \pi\left(z_{2}\right)\right)$ are Lagrangian equivalent if there exists a symplectic diffeomorphism germ $\Phi:\left(T^{*} \mathbb{R}^{n}, z_{1}\right) \rightarrow\left(T^{*} \mathbb{R}^{n}, z_{2}\right)$ with the form $\Phi(q, p)=\left(\phi_{2}(q), \phi_{1}(q, p)\right)$ and a diffeomorphism germ $\phi:\left(L_{1}, z_{1}\right) \rightarrow\left(L_{2}, z_{2}\right)$ such that $\Phi \circ \iota_{1}=\iota_{2}{ }^{\circ} \phi$.

We also have natural corresponding equivalences among the Morse families. Let $F_{i}:\left(\mathbb{R}^{k}\right.$ $\left.\times \mathbb{R}^{n}, 0\right) \rightarrow(\mathbb{R}, 0) \quad(i=1,2)$ be two Morse families. We say that $F_{1}$ and $F_{2}$ are $R^{+}$-equivalent 
(respectively, $R$-equivalent) if there exists a diffeomorphism germ $\Psi:\left(\mathbb{R}^{k} \times \mathbb{R}^{n}, 0\right) \rightarrow\left(\mathbb{R}^{k} \times \mathbb{R}^{n}, 0\right)$ with the form $\Psi(\lambda, q)=\left(\psi_{1}(\lambda, q), \psi_{2}(q)\right)$ and a function germ $\alpha:\left(\mathbb{R}^{n}, 0\right) \rightarrow \mathbb{R}$ such that $F_{1}(\lambda, q)$ $=F_{2} \circ \Psi(\lambda, q)+\alpha(q)$ (respectively, $F_{1}(\lambda, q)=F_{2} \circ \Psi(\lambda, q)$, i.e., $\alpha$ is constantly equal to 0 ). We also need the following generalized equivalence relation: For two Morse families $F_{i}:\left(\mathbb{R}^{k_{i}}\right.$ $\left.\times \mathbb{R}^{n}, 0\right) \rightarrow(\mathbb{R}, 0)(i=1,2)$, we say that $F_{1}$ and $F_{2}$ are stably $R^{+}$-equivalent if there exist nondegenerate quadratic forms $Q_{1}(\bar{\lambda}), Q_{2}(\tilde{\Lambda}),\left(\bar{\lambda} \in \mathbb{R}^{k_{3}}, \tilde{\Lambda} \in \mathbb{R}^{k_{4}}\right)$ with $k_{1}+k_{3}=k_{2}+k_{4}$ such that $F_{1}$ $+Q_{1}$ and $F_{2}+Q_{2}$ are $R^{+}$-equivalent.

The following theorem is the principal result of the Lagrangian singularity theory: ${ }^{4,5}$

Theorem 3.2: Let $F_{i}:\left(\mathbb{R}^{k} \times \mathbb{R}^{n}, 0\right) \rightarrow(\mathbb{R}, 0)(i=1,2)$ be two Morse families.

(1) If $F_{1}$ and $F_{2}$ induce the same Lagrangian submanifold germ, then $F_{1}$ and $F_{2}$ are $R$-equivalent.

(2) Lagrangian manifold germs $\Phi_{F_{1}}\left(\Sigma\left(F_{1}\right)\right)$ and $\Phi_{F_{2}}\left(\Sigma\left(F_{2}\right)\right)$ are Lagrangian equivalent if and only if $F_{1}$ and $F_{2}$ are stably $R^{+}$-equivalent.

We define the notion of stability of Lagrangian map germs as follows: A Lagrangian map germ is said to be Lagrangian stable if for every map representing the given map-germ there is a neighborhood $\mathcal{V}$ in the space of Lagrangian maps (in the $C^{\infty}$-topology) and a neighborhood $\mathcal{U}$ of the source point of the germ, such that for each Lagrangian map belonging to $\mathcal{V}$ there is a point in $\mathcal{U}$ at which the germ of Lagrangian map-germ is Lagrangian equivalent to the original germ. The corresponding infinitesimal notion for generating family is given as follows: Let $F:\left(\mathbb{R}^{k} \times \mathbb{R}^{n}, 0\right)$ $\rightarrow(\mathbb{R}, 0)$ be a Morse family. We say that $F$ is infinitesimally $R^{+}$-versal if

$$
\mathcal{E}_{\lambda}=\left\langle\frac{\partial f}{\partial \lambda_{1}}, \ldots, \frac{\partial f}{\partial \lambda_{k}}\right\rangle_{\mathcal{E}_{\lambda}}+\left\langle\frac{\partial F}{\partial q_{1}}\left|\mathbb{R}^{k} \times\{0\}, \ldots, \frac{\partial F}{\partial q_{n}}\right| \mathbb{R}^{k} \times\{0\}, 1\right\rangle_{\mathbb{R}},
$$

where $f(q)=F(q, 0)$ and $\mathcal{E}_{\lambda}$ is the local ring of function germs $\left(\mathbb{R}^{k}, 0\right) \rightarrow \mathbb{R}$. Then we have the following theorem (cf. Ref. 4).

Theorem 3.3: Let $F:\left(\mathbb{R}^{k} \times \mathbb{R}^{n}, 0\right) \rightarrow(\mathbb{R}, 0)$ be a Morse family. Then the Lagrangian map germ $\pi \circ \Phi_{F}$ is Lagrangian stable if and only if $F$ is infinitesimally $R^{+}$-versal.

Now let us recall the time-delay map $T_{\mathbf{y}}(\mathbf{x})$. If we consider the family of functions $F: \mathbb{R}^{2}$ $\times \mathbb{R}^{2} \rightarrow \mathbb{R}$ given by

$$
F\left(\lambda_{1}, \lambda_{2}, q_{1}, q_{2}\right)=\Theta\left[\frac{\left\|\left(\lambda_{1}, \lambda_{2}\right)-\left(q_{1}, q_{2}\right)\right\|^{2}}{2}-\psi\left(\lambda_{1}, \lambda_{2}\right)\right]=T_{q}(\lambda),
$$

we can easily verify that $F$ is a Morse family, so $\Phi_{F}: \Sigma(F) \rightarrow T^{*} \mathbb{R}^{2}$ is a Lagrangian immersion. Here, we have

$$
\Sigma(F)=\left\{(\mathbf{x}, \mathbf{y}) \mid \operatorname{grad}_{\mathbf{x}} T=\mathbf{0}\right\},
$$

so that the Lagrangian immersion is corresponding to those rays that are actual light rays. The set of critical values of the Lagrangian map $\pi \circ \Phi_{F}$ is the caustic.

On the other hand, we present another symplectic framework for single gravitational lensing, which is essentially the same as the above-mentioned framework. Our framework will be, however, very useful when we try to generalize this framework to the case of multiple planes gravitational lensing (cf., Sec. IV).

We consider the product symplectic space

$$
\mathcal{M}_{(x, y)}=\left(T^{*} M_{x} \times T^{*} M_{y}, \omega_{M_{y}} \ominus \omega_{M_{x}}\right),
$$

where $\omega_{M_{y}}$ and $\omega_{M_{x}}$ are the corresponding canonical symplectic forms, and $\Omega_{(x, y)}=\omega_{M_{y}} \ominus \omega_{M_{x}}$ $=\pi_{M_{y}}^{*} \omega_{M_{y}}-\pi_{M_{x}}^{*} \omega_{M_{x}}$, where $\pi_{M_{x}}, \pi_{M_{y}}$ are the canonical projections of the product 
$T^{*} M_{x} \times T^{*} M_{y}$. The corresponding phase spaces $\left(T^{*} M_{x}, \omega_{M_{x}}\right)$ and $\left(T^{*} M_{y}, \omega_{M_{y}}\right)$ are called the observer space and the source space, respectively. In our two-dimensional case $M_{x}=\mathbb{R}^{2}$ and $M_{y}=\mathbb{R}^{2}$. The concrete realized single lensing system is represented (following Ref. 6) by the Lagrangian submanifold

$$
L_{\psi}=\left\{\left(\left(\mathbf{x}, \operatorname{grad}_{\mathbf{x}} T\right),\left(\mathbf{y}, \operatorname{grad}_{\mathbf{y}} T\right)\right) \mid(\mathbf{x}, \mathbf{y}) \in M_{x} \times M_{y}\right\} .
$$

This means that the generating function of $L_{\psi}$ is the time-delay map

$$
T(\mathbf{x} ; \mathbf{y})=\Theta_{L}\left[\frac{\|\mathbf{x}-\mathbf{y}\|^{2}}{2}-\psi(\mathbf{x})\right] \quad\left(\mathbf{x}, \mathbf{y} \in \mathbb{R}^{2}\right) .
$$

By the previous arguments, light rays are given by $\left\{(\mathbf{x}, \mathbf{y}) \mid \operatorname{grad}_{\mathbf{x}} T=\mathbf{0}\right\}$ and the set of point sources for light rays is the Lagrangian submanifold

$$
L_{S}=\left\{\left(\mathbf{y}, \operatorname{grad}_{\mathbf{y}} T\right) \in T^{*} M_{y} \mid(\mathbf{x}, \mathbf{y}) \in M_{x} \times M_{y}, \operatorname{grad}_{\mathbf{x}} T=\mathbf{0}\right\}
$$

of $T^{*} M_{y}$. Then we have

$$
\left.\pi_{M_{y}}\left(L_{\psi} \cap\left(\left(M_{x} \times\{\mathbf{0}\}\right) \times T^{*} M_{y}\right)\right)\right)=L_{S} .
$$

Let us recall the basic notions of the theory of symplectic relations (Ref. 11). Let $X_{1}, X_{2}$ be smooth manifolds with the same dimension. We consider the product symplectic manifold

$$
\left(T^{*} X_{1} \times T^{*} X_{2}, \omega_{X_{2}} \ominus \omega_{X_{1}}\right),
$$

where $\omega_{X_{2}} \ominus \omega_{X_{1}}=\pi_{2}^{*} \omega_{X_{2}}-\pi_{1}^{*} \omega_{X_{1}}$. We define a symplectic relation from $T^{*} X_{1}$ to $T^{*} X_{2}$ as a Lagrangian submanifold $R$ of $\left(T^{*} X_{1} \times T^{*} X_{2}, \omega_{X_{2}} \ominus \omega_{X_{1}}\right)$. If the restriction of the projection

$$
\pi_{X_{1}} \times \pi_{X_{2}}: T^{*} X_{1} \times T^{*} X_{2} \rightarrow X_{1} \times X_{2}
$$

to $R$ is always nonsingular, we call $R$ the elementary symplectic relation. Let $R$ be a symplectic relation in $\left(T^{*} X_{1} \times T^{*} X_{2}, \omega_{X_{2}} \ominus \omega_{X_{1}}\right)$ and $S$ be a subset of $T^{*} X_{1}$, then the symplectic image of $S$ by $R$ is defined as

$$
R(S)=\left\{p_{2} \in T^{*} X_{2}: \exists_{p_{1} \in S}\left(p_{1}, p_{2}\right) \in R\right\} .
$$

If $S$ is Lagrangian submanifold in $\left(T^{*} X_{1}, \omega_{X_{1}}\right)$, then $R(S)$ is a Lagrangian subset in $\left(T^{*} X_{2}, \omega_{X_{2}}\right)$.

Since both $S$ and $R$ are Lagrangian submanifolds, we have their generating families at least locally. We only consider the local situation here, so that we assume that $X_{1}=X_{2}=\mathbb{R}^{n}$. Let $F_{1}:\left(\mathbb{R}^{k_{1}} \times X_{1}, 0\right) \rightarrow \mathbb{R}$ be a generating family of a Lagrangian submanifold germ $S \subset T^{*} X_{1}$ and $F_{2}:\left(\mathrm{R}^{k_{2}} \times\left(X_{1} \times X_{2}\right), 0\right) \rightarrow \mathrm{R}$ be a generating family of a symplectic relation $R \subset T^{*} X_{1} \times T^{*} X_{2}$. Then we have a function germ

$$
F:\left(\left(\mathbb{R}^{k_{1}} \times X_{1} \times \mathbb{R}^{k_{2}}\right) \times X_{2}, 0\right) \rightarrow \mathbb{R}
$$

defined by

$$
F\left(\left(\lambda_{1}, q_{1}, \lambda_{2}\right), q_{2}\right)=F_{1}\left(\lambda_{1}, q_{1}\right)+F_{2}\left(\lambda_{2}, q_{1}, q_{2}\right) .
$$

Hence we have the following lemma:

Lemma 3.4: If $F$ is a Morse family, then $F$ is a generating family of the Lagrangian submanifold $R(S) \subset T^{*} X_{2}$.

Proof: By definition, we have 


$$
\begin{gathered}
S=\left\{\left(q_{1}, \frac{\partial F_{1}}{\partial q_{1}}\left(\lambda_{1}, q_{1}\right)\right) \mid \frac{\partial F_{1}}{\partial \lambda_{1}}=0\right\}, \\
R=\left\{\left(q_{1}, q_{2},-\frac{\partial F_{2}}{\partial q_{1}}\left(\lambda_{2}, q_{1}\right), \frac{\partial F_{2}}{\partial q_{2}}\left(\lambda_{2}, q_{2}\right)\right) \mid \frac{\partial F_{2}}{\partial \lambda_{2}}=0\right\} .
\end{gathered}
$$

Therefore we have

$$
R(S)=\left\{\left(q_{2}, \frac{\partial F_{2}}{\partial q_{2}}\left(\lambda_{2}, q_{1}, q_{2}\right)\right) \mid \frac{\partial F_{1}}{\partial \lambda_{1}}=\frac{\partial F_{2}}{\partial \lambda_{2}}=0 \frac{\partial F_{1}}{\partial q_{1}}=-\frac{\partial F_{2}}{\partial q_{1}}\right\} .
$$

Since $\partial F / \partial \lambda_{1}=\partial F_{1} / \partial \lambda_{1}, \quad \partial F / \partial \lambda_{2}=\partial F_{2} / \partial \lambda_{2}, \quad \partial F / \partial q_{1}=\partial F_{1} / \partial q_{1}+\partial F_{2} / \partial q_{2}$ and $\partial F / \partial q_{2}$ $=\partial F_{2} / \partial q_{2}$, we have

$$
R(S)=\left\{\left(q_{2}, \frac{\partial F}{\partial q_{2}}\left(\left(\lambda_{1}, q_{1}, \lambda_{2}\right), q_{2}\right)\right) \mid \frac{\partial F}{\partial \lambda_{1}}=\frac{\partial F}{\partial \lambda_{2}}=\frac{\partial F}{\partial q_{1}}=0\right\},
$$

so that $F$ is a generating family of $R(S)$.

Q.E.D.

In the case of single gravitational lensing, if $S_{0}$ denotes the observer Lagrangian submanifold of system of gravitational rays then the source Lagrangian submanifold of rays is an image

$$
L_{\psi}\left(S_{0}\right) \subset T^{*} M_{y} .
$$

In the standard setting (cf. Ref. 6 and the previous arguments) $S_{0}$ is the zero section of the cotangent bundle $T^{*} M_{x}$. Therefore we have

$$
L_{\psi}\left(S_{0}\right)=\left\{\left(\mathbf{y}, \operatorname{grad}_{\mathbf{y}} T\right) \mid \operatorname{grad}_{\mathbf{x}} T=\mathbf{0}\right\},
$$

so that the generating family for $L_{\psi}\left(S_{0}\right)$ is given by

$$
F\left(\lambda_{1}, \lambda_{2}, q_{1}, q_{2}\right)=\Theta\left[\frac{\left\|\left(\lambda_{1}, \lambda_{2}\right)-\left(q_{1}, q_{2}\right)\right\|^{2}}{2}-\psi\left(\lambda_{1}, \lambda_{2}\right)\right],
$$

which is the same generating family as that of the source Lagrangian submanifold in the previous framework in Ref. 6. We call the pair $(S, R)$ a (single) lensing system if $S$ is a Lagrangian submanifold of $T^{*} X_{1}$ and $R$ is a symplectic relation from $T^{*} X_{1}$ to $T^{*} X_{2}$. If the projection $\left.\pi_{1}\right|_{R}: R \rightarrow T^{*} X_{1}$ is nonsingular, $R$ is the graph of a symplectomorphism $H: T^{*} M_{x} \rightarrow T^{*} M_{y}$. In this case we say that $(S, R)$ is regular. Moreover, if $S$ is the zero section of $T^{*} X_{1}$, we call $(S, R)$ a special lensing system. Therefore, the single gravitational lensing is a regular special lensing system.

\section{A SYMPLECTIC FRAMEWORK FOR MULTIPLANE GRAVITATIONAL LENSING}

In this section we will construct the intrinsic framework for the study of gravitational lensing. We can summarize the main problem in this paper as follows:

Problem: How can we construct the intrinsic framework for the study of double planes gravitational lensing?

In order to tackle this problem, we now interpret the Fermat's principle from another view point. We define

$$
T_{\beta_{23}}\left(\mathbf{x}_{2} ; \mathbf{y}\right)=\Theta_{2}\left[\frac{\left\|\mathbf{y}-\mathbf{x}_{2}\right\|^{2}}{2}-\beta_{23}\left(\mathbf{x}_{2}\right)\right],
$$




$$
T_{\beta_{12}}\left(\mathbf{x}_{1} ; \mathbf{x}_{2}\right)=\Theta_{1}\left[\frac{\left\|\mathbf{x}_{2}-\mathbf{x}_{1}\right\|^{2}}{2}-\beta_{12}\left(\mathbf{x}_{1}\right)\right]
$$

Then $T_{2}\left(\mathbf{x}_{1}, \mathbf{x}_{2}, \mathbf{y}\right)=T_{\beta_{12}}\left(\mathbf{x}_{1}, \mathbf{x}_{2}\right)+T_{\beta_{23}}\left(\mathbf{x}_{2}, \mathbf{y}\right)$. We may consider that $T_{\beta_{23}}\left(\mathbf{x}_{1}, \mathbf{x}_{2}\right)$ and $T_{\beta_{12}}\left(\mathbf{x}_{1} ; \mathbf{y}\right)$ are, respectively, single time-delay maps. It is clear that

$$
\begin{gathered}
\operatorname{grad}_{\mathbf{x}_{1}} T_{2}\left(\mathbf{x}_{1}, \mathbf{x}_{2}, \mathbf{y}\right)=\operatorname{grad}_{\mathbf{x}_{1}} T_{\beta_{12}}\left(\mathbf{x}_{1} ; \mathbf{x}_{2}\right) \\
\operatorname{grad}_{\mathbf{x}_{2}} T_{2}\left(\mathbf{x}_{1}, \mathbf{x}_{2}, \mathbf{y}\right)=\operatorname{grad}_{\mathbf{x}_{2}} T_{\beta_{12}}\left(\mathbf{x}_{1} ; \mathbf{x}_{2}\right)+\operatorname{grad}_{\mathbf{x}_{2}} T_{\beta_{23}}\left(\mathbf{x}_{2} ; \mathbf{y}\right) .
\end{gathered}
$$

These formulas suggest to us that the Fermat's principle can be interpreted as

$$
\left\{\begin{array}{l}
\operatorname{grad}_{\mathbf{x}_{1}} T_{\beta_{23}}\left(\mathbf{x}_{1}, \mathbf{x}_{2}\right)=\mathbf{0} \\
\operatorname{grad}_{\mathbf{x}_{2}} T_{2}\left(\mathbf{x}_{1}, \mathbf{x}_{2}, \mathbf{y}\right)=\mathbf{0}
\end{array}\right.
$$

It follows that we consider the following families of functions: $F_{1}\left(\mathbf{x}_{1}, \mathbf{x}_{2}\right)=T_{\beta_{12}}\left(\mathbf{x}_{1}, \mathbf{x}_{2}\right)$, $F_{2}\left(\mathbf{x}_{2}, \mathbf{y}\right)=T_{\beta_{23}}\left(\mathbf{x}_{2}, \mathbf{y}\right)$ and $F\left(\mathbf{x}_{1}, \mathbf{x}_{2}, \mathbf{y}\right)=F_{1}\left(\mathbf{x}_{1}, \mathbf{x}_{2}\right)+F_{2}\left(\mathbf{x}_{2}, \mathbf{y}\right)$. By Fermat's principle, we have two submanifolds:

$$
\begin{gathered}
\Sigma\left(F_{1}\right)=\left\{\left(\mathbf{x}_{1}, \mathbf{x}_{2}\right) \in \mathbb{R}^{4} \mid \operatorname{grad}_{\mathbf{x}_{1}} F_{1}\left(\mathbf{x}_{1}, \mathbf{x}_{2}\right)=\mathbf{0}\right\}, \\
\Sigma(F)=\left\{\left(\mathbf{x}_{1}, \mathbf{x}_{2}, \mathbf{y}\right) \in \mathbb{R}^{6} \mid \operatorname{grad}_{\mathbf{x}_{1}} F_{1}\left(\mathbf{x}_{1} ; \mathbf{x}_{2}\right)=\operatorname{grad}_{\mathbf{x}_{2}} F\left(\mathbf{x}_{1}, \mathbf{x}_{2}, \mathbf{y}\right)=\mathbf{0}\right\} .
\end{gathered}
$$

Dimensions of both submanifolds are two. Moreover, we define the following mappings:

$$
\Phi_{F_{1}}: \Sigma\left(F_{1}\right) \rightarrow T^{*} \mathbb{R}^{2}
$$

by $\Phi_{F_{1}}\left(\mathbf{x}_{1}, \mathbf{x}_{2}\right)=\left(\mathbf{x}_{2}, \operatorname{grad}_{\mathbf{x}_{2}} F_{1}\left(\mathbf{x}_{1}, \mathbf{x}_{2}\right)\right)$ and

$$
\Phi_{F}: \Sigma(F) \rightarrow T^{*} \mathbb{R}^{2}
$$

by $\Phi_{F}\left(\mathbf{x}_{1}, \mathbf{x}_{2}, \mathbf{y}\right)=\left(\mathbf{y}, \operatorname{grad}_{\mathbf{y}} F\left(\mathbf{x}_{1}, \mathbf{x}_{2}, \mathbf{y}\right)\right)$. By the previous arguments, images of both mappings are Lagrangian submanifolds. The later Lagrangian submanifold corresponds to the light source. The first Lagrangian submanifold corresponds to the light ray image on the second lens, since the distance between the first lens and the second lens is so long that there might be no interactions between these lenses. So we have to consider the stability of light ray under the independent perturbations of each lens planes.

On the other hand, we might consider that $F_{2}\left(\mathbf{x}_{2}, \mathbf{y}\right)$ is a generating function of the graph of a certain symplectomorphism $H: T^{*} \mathbb{R}^{2} \rightarrow T^{*} \mathrm{R}^{2}$ (cf., Ref. 11). In this case, the Lagrangian submanifold $\Phi_{F}(\Sigma(F))$ can be considered as the image $H\left(\Phi_{F_{1}}\left(\Sigma\left(F_{1}\right)\right)\right)$.

Since the single lensing system can be described under the framework of symplectic relations, we might construct the framework for the double gravitation lensing by using the composition of two symplectic relations.

The composition $R_{2} \circ R_{1} \subset\left(T^{*} X_{1} \times T^{*} X_{3}, \omega_{X_{3}} \ominus \omega_{X_{1}}\right)$ of two symplectic relations $R_{1} \subset\left(T^{*} X_{1} \times T^{*} X_{2}, \omega_{X_{2}} \ominus \omega_{X_{1}}\right), R_{2} \subset\left(T^{*} X_{2} \times T^{*} X_{3}, \omega_{X_{3}} \ominus \omega_{X_{2}}\right)$ is defined as follows:

$$
R_{2} \circ R_{1}=\left\{\left(p_{1}, p_{3}\right) \in T^{*} X_{1} \times T^{*} X_{3} ; \exists_{p_{2} \in T^{*} X_{2}}\left(p_{1}, p_{2}\right) \in R_{1} \text { and }\left(p_{2}, p_{3}\right) \in R_{2}\right\}
$$

If $S$ is Lagrangian submanifold in $T^{*} X_{1}$, then we have two symplectic images

$$
R_{1}(S) \text { and } R_{2} \circ R_{1}(S)
$$


By the previous arguments, a double gravitational lensing is represented by the pair of symplectic relations $\left(L_{\beta_{12}}, L_{\beta_{23}}\right)$,

$$
\begin{gathered}
L_{\beta_{12}} \subset\left(T^{*} M_{x_{1}} \times T^{*} M_{x_{2}}, \omega_{M_{r_{2}}} \ominus \omega_{M_{x_{1}}}\right), \\
L_{\beta_{23}} \subset\left(T^{*} M_{x_{2}} \times T^{*} M_{y}, \omega_{M_{y}} \ominus \omega_{M_{x_{2}}}\right) .
\end{gathered}
$$

Now the source Lagrangian subspace of the system is the image by the composition; $L_{\beta_{23}}$ ${ }^{\circ} L_{\beta_{12}}\left(S_{0}\right)$, where $S_{0}$ is the zero section of $T^{*} M_{x_{1}}$. Since $T_{\beta_{12}}\left(\mathbf{x}_{1}, \mathbf{x}_{2}\right)$ and $T_{\beta_{23}}\left(\mathbf{x}_{2}, \mathbf{y}\right)$ are the corresponding generating functions for the symplectic relations (the graphs of symplectomorphism) $L_{\beta_{12}}$ and $L_{\beta_{23}}$, then the configurational positions of rays emitted from the source at a point $\mathbf{y}$ are the points $\left(\left(\mathbf{x}_{1}, \mathbf{x}_{2}\right),\left(\mathbf{x}_{2}, \mathbf{y}\right)\right) \in L_{\beta_{12}} \times L_{\beta_{23}}$ given by the solutions $\left(\mathbf{x}_{1}, \mathbf{x}_{2}\right)$ of the system of equations

$$
\begin{aligned}
& \operatorname{grad}_{\mathbf{x}_{1}} T_{2}\left(\mathbf{x}_{1}, \mathbf{x}_{2}, \mathbf{y}\right)=\mathbf{0}, \\
& \operatorname{grad}_{\mathbf{x}_{2}} T_{2}\left(\mathbf{x}_{1}, \mathbf{x}_{2}, \mathbf{y}\right)=\mathbf{0} .
\end{aligned}
$$

If we now consider a family of functions $F: \mathbb{R}^{2} \times \mathbb{R}^{2} \times M_{y} \rightarrow \mathbb{R}$ defined by

$$
F(q, \lambda, \mu)=T_{2}(q, \lambda, \mu)=T_{\beta_{12}}(q, \lambda)+T_{\beta_{23}}(\lambda, \mu),
$$

then $F$ is a generating family for the image Lagrangian subspace

$$
L_{\beta_{23}}{ }^{\circ} L_{\beta_{12}}\left(S_{0}\right) \subset T^{*} M_{y} .
$$

According to the above-given arguments, we say that $\left(S, R_{1}, R_{2}\right)$ is a double lensing system if $S$ is a Lagrangian submanifold of $T^{*} X_{1}$ and $R_{i}$ is a symplectic relation from $T^{*} X_{i}$ to $T^{*} X_{i+1}$, where $i=1,2$. We also say that the double lensing system $\left(S, R_{1}, R_{2}\right)$ is regular if both of $R_{i}(i$ $=1,2$ ) are graphs of symplectomorphisms. Moreover, we say that a double lensing system $\left(S, R_{1}, R_{2}\right)$ is special if $S$ is the zero section of $T^{*} X_{1}$. Therefore, a double gravitation lensing is a regular special double lensing system.

We now define a natural equivalence relation among double lensing system germs. Let $\left(S, R_{1}, R_{2}\right),\left(S^{\prime}, R_{1}^{\prime}, R_{2}^{\prime}\right)$ be double lensing system germs. We say that $\left(S, R_{1}, R_{2}\right),\left(S^{\prime}, R_{1}^{\prime}, R_{2}^{\prime}\right)$ are Lagrangian equivalent if there exist a symplectic diffeomorphism germ and

$$
\Phi_{1}:\left(T^{*} X_{1}, z_{1}\right) \rightarrow\left(T^{*} X_{1}, z_{1}^{\prime}\right),
$$

Lagrangian equivalence germs (symplectic diffeomorphisms preserving the cotangent bundle fibration)

$$
\Phi_{2}:\left(T^{*} X_{2}, z_{2}\right) \rightarrow\left(T^{*} X_{2}, z_{2}^{\prime}\right), \quad \Phi_{3}:\left(T^{*} X_{3}, z_{3}\right) \rightarrow\left(T^{*} X_{3}, z_{3}^{\prime}\right)
$$

such that $\Phi_{1}(S)=S^{\prime},\left(\Phi_{1} \times \Phi_{2}\right)\left(R_{1}\right)=R_{1}^{\prime}$ and $\left(\Phi_{2} \times \Phi_{3}\right)\left(R_{2}\right)=R_{2}^{\prime}$.

Since there always exists a symplectic diffeomorphism germ $\Phi_{1}:\left(T^{*} X_{1}, z_{1}\right) \rightarrow\left(T^{*} X_{1}, z_{1}^{\prime}\right)$ such that $\Phi_{1}(S)=S^{\prime}$, we may assume that $S=S^{\prime}$ are equal to the zero section of $T^{*} X_{1}$. Therefore, without the loss of generality, we stick to special double lensing. In this case we say that $\left(S, R_{1}, R_{2}\right),\left(S, R_{1}^{\prime}, R_{2}^{\prime}\right)$ are strictly Lagrangian equivalent if there exist Lagrangian equivalence germs

$$
\Phi_{2}:\left(T^{*} X_{2}, z_{2}\right) \rightarrow\left(T^{*} X_{2}, z_{2}^{\prime}\right), \quad \Phi_{3}:\left(T^{*} X_{3}, z_{3}\right) \rightarrow\left(T^{*} X_{3}, z_{3}^{\prime}\right)
$$

such that $\left(\mathrm{id}_{T * X_{1}} \times \Phi_{2}\right)\left(R_{1}\right)=R_{1}^{\prime}$ and $\left(\Phi_{2} \times \Phi_{3}\right)\left(R_{2}\right)=R_{2}^{\prime}$. This equivalence relation might be, however, too strong to give a classification of double lensing system germs. Therefore, we need an appropriate equivalence relation among special double lensing system germs. 
Now we give a candidate of such a natural equivalence. We say that the special double lensing system germs $\left(S, R_{1}, R_{2}\right),\left(S, R_{1}^{\prime}, R_{2}^{\prime}\right)$ are weakly Lagrangian equivalent if there exist Lagrangian equivalence germs

$$
\tilde{\Phi}_{3}:\left(T^{*} X_{3}, z_{3}\right) \rightarrow\left(T^{*} X_{3}, z_{3}^{\prime}\right), \quad \tilde{\Phi}:\left(\left(T^{*} X_{2} \times T^{*} X_{3},\left(z_{2}, z_{3}\right)\right) \rightarrow\left(T^{*} X_{2} \times T^{*} X_{3},\left(z_{2}^{\prime}, z_{3}^{\prime}\right)\right)\right.
$$

such that $\pi_{X_{3}} \circ \widetilde{\Phi}=\widetilde{\Phi}_{3} \circ \pi_{X_{3}}$ and $\widetilde{\Phi}\left(R_{1}(S) \times R_{2} \circ R_{1}(S)\right)=R_{1}^{\prime}(S) \times R_{2}^{\prime} \circ R_{1}^{\prime}(S)$.

We have the following proposition.

Proposition 4.1: Suppose that $\left(S, R_{1}, R_{2}\right),\left(S, R_{1}^{\prime}, R_{2}^{\prime}\right)$ are weakly Lagrangian equivalent, then both of the Lagrangian submanifolds $R_{1}(S), R_{1}^{\prime}(S)$ and $R_{2}^{\circ} R_{1}(S), R_{2}^{\prime} \circ R_{2}^{\prime}(S)$ are Lagrangian equivalent.

Proof: By definition we have a diffeomorphism germ $\Phi:\left(X_{2} \times X_{3},\left(\pi_{2}\left(z_{2}\right), \pi_{3}\left(z_{3}\right)\right)\right) \rightarrow\left(X_{2}\right.$ $\left.\times X_{3},\left(\pi_{2}\left(z_{2}^{\prime}\right), \pi_{3}\left(z_{3}^{\prime}\right)\right)\right)$ of the form $\Phi\left(x_{2}, x_{3}\right)=\left(\phi_{2}\left(x_{2}, x_{3}\right), \phi_{3}\left(x_{3}\right)\right)$ and a symplectic diffeomorphism $\widetilde{\Phi}:\left(\left(T^{*} X_{2} \times T^{*} X_{3},\left(z_{2}, z_{3}\right)\right) \rightarrow\left(T^{*} X_{2} \times T^{*} X_{3},\left(z_{2}^{\prime}, z_{3}^{\prime}\right)\right)\right.$ of the form

$$
\widetilde{\Phi}\left(\left(x_{2}, p_{2}\right),\left(x_{3}, p_{3}\right)\right)=\left(\left(\phi_{2}\left(x_{2}, x_{3}\right), \psi_{2}\left(x_{2}, x_{3}, p_{2}, p_{3}\right)\right), \phi_{3}\left(x_{3}\right), \psi_{3}\left(x_{3}, p_{3}\right)\right)
$$

such that $\tilde{\Phi}\left(R_{1}(S) \times R_{2} \circ R_{3}(S)\right)=R_{1}^{\prime}(S) \times R_{2}^{\prime} \circ R_{1}^{\prime}(S)$. Therefore we have $\tilde{\Phi}\left(R_{1}(S) \times\left\{z_{3}\right\}\right)$ $=\left(R_{1}^{\prime}(S) \times\left\{z_{3}^{\prime}\right\}\right)$. We identify symplectic manifolds: $T^{*} X_{2}=T^{*} X_{2} \times\left\{z_{3}\right\}=T^{*} X_{2} \times\left\{z_{3}^{\prime}\right\}$. Under this identification, the above-mentioned equality means that $R_{1}(S)$ and $R_{1}^{\prime}(S)$ are Lagrangian equivalent.

By definition, we have $\widetilde{\Phi}_{3}\left(R_{2}^{\circ} R_{1}(S)\right)=R_{2}^{\prime} \circ R_{1}^{\prime}(S)$. This fact means that $R_{2}^{\circ} R_{1}(S), R_{2}^{\prime}$ $\circ R_{2}^{\prime}(S)$ are Lagrangian equivalent.

Q.E.D.

By the above-given proposition, the weak Lagrangian equivalence among double lensing system germs preserve both caustics of the first and the second deflectors. It is the caustic equivalence already introduced in the classification of coisotropic varieties in Ref. 12.

\section{GENERATING PAIRS FOR DOUBLE LENSING SYSTEMS}

In this section we consider the problem how to construct a kind of the notion of generating families for double lensing systems. We already have a solution because a double plane gravitational lensing is described by the pair of time-delay maps. We only consider local properties, so that we assume that $X_{1}=X_{2}=X_{3}=\mathbb{R}^{n}$.

For any double lensing system germ $\left(S, R_{1}, R_{2}\right)$, we have generating families $F_{0}:\left(\mathbb{R}^{k_{0}}\right.$ $\left.\times X_{1}, 0\right) \rightarrow \mathbb{R}$ of $S, F_{1}:\left(\mathbb{R}^{k_{1}} \times\left(X_{1} \times X_{2}\right), 0\right) \rightarrow \mathbb{R}$ of $R_{1}$ and $F_{2}:\left(\mathbb{R}^{k_{2}} \times\left(X_{2} \times X_{3}\right), 0\right) \rightarrow \mathbb{R}$ of $R_{2}$.

On the other hand, there always exists a symplectomorphism germ $\Phi_{1}:\left(T^{*} X_{1}, z_{1}\right)$ $\rightarrow\left(T^{*} X_{1}, 0\right)$ such that $\Phi_{1}(S)$ is a zero section germ of $T^{*} X_{1}$, so that we might assume that $S$ is a zero section germ of $T^{*} X_{1}$ under the Lagrangian equivalence among double lensing system germs. In other words, it is enough to investigate special double lensing system germs. In this case $F_{0}$ can be chosen as a constant function. We call $\left(F_{1}, F_{2}\right)$ a generating pair of the special lensing system germ $\left(S, R_{1}, R_{2}\right)$ if $F_{i}$ is a generating family of $R_{i}(i=1,2)$. Then $F_{1}$ can be regarded as a generating family of $R_{1}(S) \subset T^{*} X_{2}$ and $F=F_{1}+F_{2}$ is a generating family of $R_{2}^{\circ} R_{1}(S) \subset T^{*} X_{3}$ (cf., Ref. 11).

Since a double gravitation lensing is a regular double lensing system, we now pay attention to regular special double lensing systems here. In this case $F_{1}:\left(X_{1} \times X_{2}, 0\right) \rightarrow \mathbb{R}$ is a generating function of $R_{1}$ and $F_{2}:\left(X_{2} \times X_{3}, 0\right) \rightarrow \mathbb{R}$ is a generating function of $R_{2}$. By the arguments in the previous paragraph, $F_{1}$ is a generating family of the Lagrangian submanifold germ $R_{1}(S) \subset T^{*} X_{2}$ and a map germ $F:\left(\left(X_{1} \times X_{2}\right) \times X_{3}, 0\right) \rightarrow \mathrm{R}$ defined by

$$
F\left(\left(x_{1}, x_{2}\right), x_{3}\right)=F_{1}\left(x_{1}, x_{2}\right)+F_{2}\left(x_{2}, x_{3}\right)
$$


is a generating family of the Lagrangian submanifold germ $R_{2} \circ R_{1}(S) \subset T^{*} X_{3}$. In other words, $\left(F_{1}, F_{2}\right)$ is a generating pair of a regular special double lensing system germ $\left(S, R_{1}, R_{2}\right)$ if $\left(d F_{1}\left(X_{1} \times X_{2}\right),\left(z_{1}, z_{2}\right)\right)=\left(R_{1},\left(z_{1}, z_{2}\right)\right)$ and $\left(d F_{2}\left(X_{2} \times X_{3}\right),\left(z_{2}, z_{3}\right)\right)=\left(R_{2},\left(z_{2}, z_{3}\right)\right)$.

Since any elementary symplectic relation has a generating function at least locally, we have the following fundamental proposition:

Proposition 5.1: All regular special double lensing system germs are constructed by the above-mentioned method.

We can translate equivalence relations among double lensing systems into those of corresponding generating pairs. We consider the ambiguity of the choice for generating pairs of a double lensing system.

Proposition 5.2: Let $\left(F_{1}, F_{2}\right)$ and $\left(G_{1}, G_{2}\right)$ be generating pairs of a common regular special double lensing system germ. Then $F_{1}=G_{1}+$ constant and $F_{2}=G_{2}+$ constant.

For our purpose, we introduce equivalence relations among generating pairs for double lensing system germs. Let $F_{1}, G_{1},\left(X_{1} \times X_{2}, 0\right) \rightarrow \mathbb{R}$ and $F_{2}, G_{2},\left(X_{2} \times X_{3}, 0\right) \rightarrow \mathbb{R}$ be function germs. We say that $\left(F_{1}, F_{2}\right)$ and $\left(G_{1}, G_{2}\right)$ are $(R, L)^{+}$-equivalent if there exist diffeomorphism germs $\Phi_{1}:\left(X_{1} \times X_{2}, 0\right) \rightarrow\left(X_{1} \times X_{2}, 0\right)$ of the form $\Phi_{1}\left(x_{1}, x_{2}\right)=\left(\phi_{1}\left(x_{1}, x_{2}\right), \phi_{2}\left(x_{2}\right)\right)$, and $\Phi_{2}:\left(X_{2}\right.$ $\left.\times X_{3}, 0\right) \rightarrow\left(X_{2} \times X_{3}, 0\right)$ of the form $\Phi_{2}\left(x_{2}, x_{3}\right)=\left(\phi_{2}\left(x_{2}\right), \phi_{3}\left(x_{3}\right)\right)$ and function germs $\alpha:\left(X_{2}, 0\right)$ $\rightarrow \mathbb{R}, \beta:\left(X_{3}, 0\right) \rightarrow \mathbb{R}$ such that

$$
\left\{\begin{array}{l}
F_{1}\left(x_{1}, x_{2}\right)=G_{1} \circ \Phi_{1}\left(x_{1}, x_{2}\right)+\alpha\left(x_{2}\right), \\
F_{2}\left(x_{2}, x_{3}\right)=G_{2} \circ \Phi_{2}\left(x_{2}, x_{3}\right)+\beta\left(x_{3}\right) .
\end{array}\right.
$$

Proposition 5.3: Let $\left(F_{1}, F_{2}\right)$ and $\left(G_{1}, G_{2}\right)$ be, respectively, generating pairs of special regular double lensing system germs $\left(S_{0}, R_{1}, R_{2}\right)$ and $\left.\left(S_{0}, R_{1}^{\prime}, R_{2}^{\prime}\right)\right)$. Then $\left(S_{0}, R_{1}, R_{2}\right)$ and $\left.\left(S_{0}, R_{1}^{\prime}, R_{2}^{\prime}\right)\right)$ are strictly Lagrangian equivalent if and only if $\left(F_{1}, F_{2}\right)$ and $\left(G_{1}, G_{2}\right)$ are $(R, L)^{+}$-equivalent.

We also say that $\left(F_{1}, F_{2}\right)$ and $\left(G_{1}, G_{2}\right)$ are $R \times L^{+}$-equivalent if there exists a diffeomorphism germ

$$
\Phi:\left(X_{1} \times X_{2} \times X_{3}, 0\right) \rightarrow\left(X_{1} \times X_{2} \times X_{3}, 0\right)
$$

of the form $\Phi\left(x_{1}, x_{2}, x_{3}\right)=\left(\phi_{1}\left(x_{1}, x_{2}, x_{3}\right), \phi_{2}\left(x_{2}, x_{3}\right), \phi\left(x_{3}\right)\right)$ and a function germ $\alpha:\left(X_{3}, 0\right) \rightarrow \mathbb{R}$ such that

$$
F_{1}\left(x_{1}, x_{2}\right)+F_{2}\left(x_{2}, x_{3}\right)=G_{1}\left(\phi_{1}\left(x_{1}, x_{2}, x_{3}\right), \phi_{2}\left(x_{2}, x_{3}\right)\right)+G_{2}\left(\phi_{2}\left(x_{2}, x_{3}\right), \phi\left(x_{3}\right)\right)+\alpha\left(x_{3}\right) .
$$

Suppose that $\left(F_{1}, F_{2}\right)$ and $\left(G_{1}, G_{2}\right)$ are $R \times L^{+}$-equivalent. If we substitute $x_{3}=0$ into the both sides of the above-given equality, then we have

$$
F_{1}\left(x_{1}, x_{2}\right)+F_{2}\left(x_{2}, 0\right)=G_{1}\left(\phi_{1}\left(x_{1}, x_{2}, 0\right), \phi_{2}\left(x_{2}, 0\right)\right)+G_{2}\left(\phi_{2}\left(x_{2}, 0\right), 0\right)+\alpha(0) .
$$

Therefore, $F_{1}$ and $G_{1}$ are $R^{+}$-equivalent.

By the general theory for Lagrangian singularities (cf., Ref. 4), we can show the following proposition:

Proposition 5.4: Let $\left(F_{1}, F_{2}\right)$ and $\left(G_{1}, G_{2}\right)$ be, respectively, generating pairs of special regular double lensing system germs $\left(S_{0}, R_{1}, R_{2}\right)$ and $\left.\left(S_{0}, R_{1}^{\prime}, R_{2}^{\prime}\right)\right)$. Then $\left(S_{0}, R_{1}, R_{2}\right)$ and $\left.\left(S_{0}, R_{1}^{\prime}, R_{2}^{\prime}\right)\right)$ are weakly Lagrangian equivalent if and only if $\left(F_{1}, F_{2}\right)$ and $\left(G_{1}, G_{2}\right)$ are $R$ $\times L^{+}$-equivalent.

We say that $\left(F_{1}, F_{2}\right)$ is infinitesimally $R \times L^{+}$-stable if

$$
\mathcal{E}_{\left(x_{1}, x_{2}\right)}+\mathcal{E}_{\left(x_{2}, x_{3}\right)} \subset\left\langle\frac{\partial F_{1}}{\partial x_{1}}\right\rangle_{\mathcal{E}_{\left(x_{1}, x_{2}, x_{3}\right)}}+\left\langle\frac{\partial\left(F_{1}+F_{2}\right)}{\partial x_{2}}\right\rangle_{\mathcal{E}_{\left(x_{2}, x_{3}\right)}}+\left\langle\frac{\partial F_{2}}{\partial x_{3}}, 1\right\rangle_{\mathcal{E}_{x_{3}}} .
$$

In this case, for $F_{1}$ we have 


$$
\mathcal{E}_{x_{1}}=\left\langle\frac{\partial f_{1}}{\partial x_{1}}\right\rangle_{\mathcal{E}_{x_{1}}}+\left\langle\left.\frac{\partial F_{1}}{\partial x_{2}}\right|_{x_{2}=0}, 1\right\rangle_{\mathbb{R}}
$$

where $f_{1}\left(x_{1}\right)=F_{1}\left(x_{1}, 0\right)$.

Now we have the following proposition:

Proposition 5.5: Let $\left(F_{1}, F_{2}\right)$ be a generating pair of a special regular double lensing system germ $\left(S_{0}, R_{1}, R_{2}\right)$. Then the following are equivalent:

(1) $\left(F_{1}, F_{2}\right)$ is infinitesimally $R \times L^{+}$-stable.

(2) $\left(F_{1}, F_{2}\right)$ satisfies the following condition:

$$
\mathcal{E}_{\left(x_{1}, x_{2}\right)}=\left\langle\frac{\partial F_{1}}{\partial x_{1}}\right\rangle_{\mathcal{E}_{\left(x_{1}, x_{2}\right)}}+\left\langle\frac{\partial\left(F_{1}+f_{2}\right)}{\partial x_{2}}\right\rangle_{\mathcal{E}_{x_{2}}}+\left\langle\left.\frac{\partial F_{2}}{\partial x_{3}}\right|_{x_{3}=0}, 1\right\rangle_{\mathbb{R}},
$$

where $f_{2}\left(x_{2}\right)=F_{2}\left(x_{2}, 0\right)$.

(3) $\left(F_{1}, F_{2}\right)$ satisfies the following condition:

$$
\mathcal{E}_{\left(x_{1}, x_{2}\right)}+\mathcal{E}_{\left(x_{2}, x_{3}\right)}=\left\langle\frac{\partial F_{1}}{\partial x_{1}}\right\rangle_{\mathcal{E}_{\left(x_{1}, x_{2}, x_{3}\right)}}+\left\langle\frac{\partial\left(F_{1}+F_{2}\right)}{\partial x_{2}}\right\rangle_{\mathcal{E}_{\left(x_{2}, x_{3}\right)}}+\left\langle\frac{\partial F_{2}}{\partial x_{3}}, 1\right\rangle_{\mathcal{E}_{x_{3}}} .
$$

(4) $F_{1}$ is infinitesimally $R^{+}$-versal and

$$
\mathcal{E}_{x_{2}} \subset\left\langle\frac{\partial F_{1}}{\partial x_{1}}\right\rangle_{\mathcal{E}_{\left(x_{1}, x_{2}\right)}}+\left\langle\frac{\partial\left(F_{1}+f_{2}\right)}{\partial x_{2}}\right\rangle_{\mathcal{E}_{x_{2}}}+\left\langle\left.\frac{\partial F_{2}}{\partial x_{3}}\right|_{x_{3}=0}, 1\right\rangle_{\mathbb{R}} .
$$

Proof: We assume that the condition (4) holds. Since $F_{1}$ is infinitesimally $R^{+}$-versal deformation of $f_{1}$, we can show that

$$
\mathcal{E}_{\left(x_{1}, x_{2}\right)}=\left\langle\frac{\partial F_{1}}{\partial x_{1}}\right\rangle_{\mathcal{E}_{\left(x_{1}, x_{2}\right)}}+\left\langle\frac{\partial F_{1}}{\partial x_{2}}, 1\right\rangle_{\mathcal{E}_{x_{2}}}
$$

We remark that

$$
\frac{\partial F_{1}}{\partial x_{2}}=\frac{\partial\left(F_{1}+f_{2}\right)}{\partial x_{2}}-\frac{\partial f_{2}}{\partial x_{2}}
$$

so that we have

$$
\mathcal{E}_{\left(x_{1}, x_{2}\right)}=\left\langle\frac{\partial F_{1}}{\partial x_{1}}\right\rangle_{\mathcal{E}_{\left(x_{1}, x_{2}\right)}}+\left\langle\frac{\partial\left(F_{1}+f_{2}\right)}{\partial x_{2}}\right\rangle_{\mathcal{E}_{x_{2}}}+\left\langle\left.\frac{\partial F_{2}}{\partial x_{3}}\right|_{x_{3}=0}, 1\right\rangle_{\mathrm{R}}+\mathcal{E}_{x_{2}} .
$$

It follows from the assumption that

$$
\mathcal{E}_{\left(x_{1}, x_{2}\right)}=\left\langle\frac{\partial F_{1}}{\partial x_{1}}\right\rangle_{\mathcal{E}_{\left(x_{1}, x_{2}\right)}}+\left\langle\frac{\partial\left(F_{1}+f_{2}\right)}{\partial x_{2}}\right\rangle_{\mathcal{E}_{x_{2}}}+\left\langle\left.\frac{\partial F_{2}}{\partial x_{3}}\right|_{x_{3}=0}, 1\right\rangle_{\mathbb{R}} .
$$

This means that the condition (2) holds. The converse assertion is trivial by definition.

By the Malgrange preparation theorem (cf., Ref. 13), we can easily show that the condition (3) is equivalent to the condition (2). Since $\mathcal{E}_{\left(x_{1}, x_{2}\right)}+\mathcal{E}_{x_{2}}=\mathcal{E}_{\left(x_{1}, x_{2}\right)}$, condition (1) implies condition (2). It follows from the inclusion $\mathcal{E}_{\left(x_{1}, x_{2}\right)}+\mathcal{E}_{\left(x_{2}, x_{3}\right)} \subset \mathcal{E}_{\left(x_{1}, x_{2}, x_{3}\right)}$ that condition (3) implies condition (1). This completes the proof. ${ }^{14}$ 


\section{CLASSIFICATION OF LENSING SYSTEMS AND CAUSTICS}

We recall that the family of functions

$$
F\left(x_{1}, x_{2}, x_{3}\right)=F_{1}\left(x_{1}, x_{2}\right)+F_{2}\left(x_{2}, x_{3}\right)
$$

is a generating family for the Lagrangian submanifold-germ $R_{2}^{\circ} R_{1}(S) \subset T^{*} X_{3}$ with $\left(x_{1}, x_{2}\right)$ being the Morse parameters auxiliary in the reduction process (cf. Ref. 15). A caustic of the time-delay map in the case of the double lensing system is defined to be the set of source positions $x_{3}$ $\in \mathbb{R}^{2}$, which are critical values of the projection

$$
T^{*} X_{3} \ni R_{2} \circ R_{1}(S) \rightarrow X_{3},
$$

or the function $\left(x_{1}, x_{2}\right) \rightarrow F\left(x_{1}, x_{2}, x_{3}\right)$ has at least one degenerate critical point.

Using the $R \times L^{+}$equivalency group and infinitesimal stability conditions obtained in Propositions 5.4 and 5.5 we can construct normal and prenormal forms of infinitesimally stable generating pairs $\left(F_{1}, F_{2}\right)$, or equivalently the functions $F\left(x_{1}, x_{2}, x_{3}\right)=F_{1}\left(x_{1}, x_{2}\right)+F_{2}\left(x_{2}, x_{3}\right)$, which belong to the space of additively composed functions on $\Delta=\left\{\left(x_{1}, x_{2}, \tilde{x}_{2}, x_{3}\right) \in X_{1} \times X_{2} \times X_{2}\right.$ $\left.\times X_{3}: x_{2}=\tilde{x}_{2}\right\}$, i.e.,

$$
F\left(x_{1}, x_{2}, x_{3}\right)=\pi_{12}^{*} F_{1}\left(x_{1}, x_{2}\right)+\left.\pi_{23}^{*} F_{2}\left(\tilde{x}_{2}, x_{3}\right)\right|_{\Delta},
$$

where $\pi_{12}, \pi_{23}$ are the canonical projections

$$
\pi_{i j}: X_{1} \times X_{2} \times X_{2} \times X_{3} \rightarrow X_{i} \times X_{j}, \quad(i j)=(12),(23) .
$$

If $\left(F_{1}, F_{2}\right)$ is an infinitesimally $R \times L^{+}$-stable generating pair, then by Proposition 5.5 (4) $F_{1}$ is infinitesimally versal. Now we define the subgroup of $R \times L^{+}$-equivalency group prescribed to $F_{1}$ and acting on $F_{2}$. We say that $F_{2}$ and $G_{2}$ are $\left(R \times L^{+}\right)_{F_{1}}$ equivalent if there exists $\Phi\left(x_{1}, x_{2}, x_{3}\right)=\left(\phi_{1}\left(x_{1}, x_{2}, x_{3}\right), \phi_{2}\left(x_{2}, x_{3}\right), \phi\left(x_{3}\right)\right)$ and a function-germ $\alpha:\left(X_{3}, 0\right) \rightarrow R$ such that $F_{1}$ is preserved, $F_{1} \circ\left(\phi_{1}, \phi_{2}\right)=F_{1}$ and $\left(F_{1}, F_{2}\right)$ and $\left(F_{1}, G_{2}\right)$ are $R \times L^{+}$-equivalent by $\Phi$. Now we can formulate the following result.

Proposition 6.1: Any infinitesimally $R \times L^{+}$-stable pair is $R \times L^{+}$-equivalent to the pair $\left(F_{1}, F_{2}\right)$, where $F_{1}$ is a versal unfolding of $F_{1}\left(x_{1}, 0\right)$ and $F_{2}$ belongs to an open orbit of $(R$ $\left.\times L^{+}\right)_{F_{1}}$-action.

In the two-dimensional case we simplify the notation and write

$$
F(x, u, v)=F_{1}(x, u)+F_{2}(u, v), \quad x, u, v \in \mathbb{R}^{2} .
$$

Proposition 6.2: Let us assume that $F_{1}$ is an infinitesimally $R^{+}$-versal Morse family. Then the generic generating pair-germs $\left(F_{1}, F_{2}\right)$ are $R \times L^{+}$-equivalent to one of the following normal forms:

$$
\begin{gathered}
\left(A_{1} A_{1}\right): \quad\left(F_{1}(x, u), F_{2}(u, v)\right)=\left( \pm x_{1}^{2} \pm x_{2}^{2}, \pm u_{1}^{2} \pm u_{2}^{2}\right), \\
\left(A_{1} A_{2}\right): \quad\left(F_{1}(x, u), F_{2}(u, v)\right)=\left( \pm x_{1}^{2} \pm x_{2}^{2}, u_{1}^{3} \pm u_{2}^{2}+v_{1} u_{1}\right), \\
\left(A_{1} A_{3}\right): \quad\left(F_{1}(x, u), F_{2}(u, v)\right)=\left( \pm x_{1}^{2} \pm x_{2}^{2}, \pm u_{1}^{4} \pm u_{2}^{2}+v_{1} u_{1}^{2}+v_{2} u_{1}\right), \\
\left(A_{2} B_{2}\right): \quad\left(F_{1}(x, u), F_{2}(u, v)\right)=\left(x_{1}^{3} \pm x_{2}^{2}+x_{1} u_{1}, \pm u_{1}^{2} \pm u_{2}^{2}+v_{1} u_{1}\right), \\
\left(A_{2} B_{3}\right): \quad\left(F_{1}(x, u), F_{2}(u, v)\right)=\left(x_{1}^{3} \pm x_{2}^{2}+x_{1} u_{1}, u_{1}^{3} \pm u_{2}^{2}+v_{1} u_{1}+v_{2} u_{1}^{2}\right), \\
\left(A_{2} C_{2}\right): \quad\left(F_{1}(x, u), F_{2}(u, v)\right)=\left(x_{1}^{3} \pm x_{2}^{2}+x_{1} u_{1}, u_{1} u_{2} \pm u_{2}^{2}+v_{1} u_{2}\right),
\end{gathered}
$$




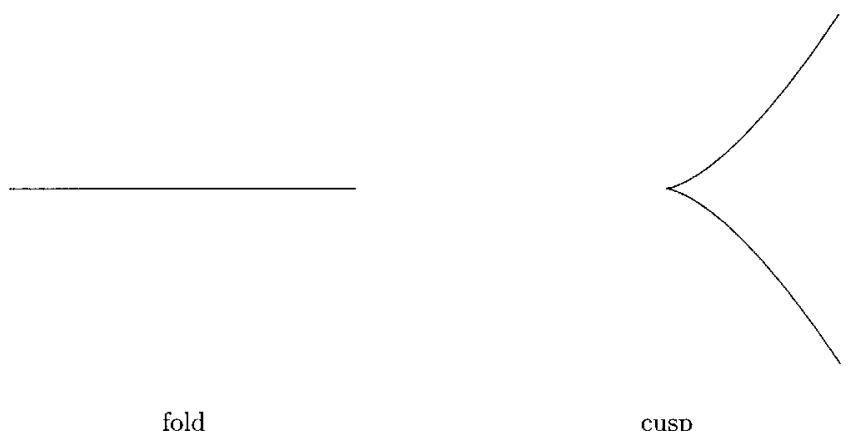

FIG. 3. The standard plane gravitational lensing.

$$
\begin{gathered}
\left(A_{2} C_{3}\right): \quad\left(F_{1}(x, u), F_{2}(u, v)\right)=\left(x_{1}^{3} \pm x_{2}^{2}+x_{1} u_{1}, u_{1} u_{2}+u_{2}^{3}+v_{1} u_{2}+v_{2} u_{2}^{2}\right), \\
\left(A_{3} X\right): \quad\left(F_{1}(x, u), F_{2}(u, v)\right)=\left( \pm x_{1}^{4} \pm x_{2}^{2}+x_{1}^{2} u_{1}+x_{1} u_{2}, \xi(u, v)\right)
\end{gathered}
$$

where $\xi$ is a smooth function-germ.

The first three cases $(A A)$ give the standard plane caustics (i.e., nonsingular, folds and cusps).

The four cases $(A B),(A C)$ are the composed $A, B$, and $C$ boundary type caustics. However, only the case $\left(\mathrm{A}_{2} \mathrm{C}_{3}\right)$ has the caustics at the origin as a composition. In this case we can calculate that (Fig. 3)

$$
R_{2}^{\circ} R_{1}(s)=\left\{\left(-2 x y, y, x, x^{2}\right) \mid(x, y) \in\left(\mathbb{R}^{2}, 0\right)\right\} \subset T^{*} X_{3} .
$$

Therefore the projection onto $X_{3}$ is locally represented by $f:\left(\mathbb{R}^{2}, 0\right) \rightarrow\left(\mathbb{R}^{2}, 0\right) ; f(x, y)=$ $(-2 x y, y)$ This map-germ is called the pinch map (cf., Fig. 4). This is a famous example which does not admit a Thom stratification (Ref. 16, p. 24).

The last case gives other possibilities of compositions with $A_{3}$-caustic. There might appear several complicated singularities.

An equivalence $R \times L^{+}$-group acting on $X_{1} \times X_{2} \times X_{3}$ is a subgroup of the $(r, s)$-equivalences introduced in Ref. 17, where $r=\operatorname{dim} X_{2}$ and $s=\operatorname{dim} X_{3}$. We recall that $(r, s)$-infinitesimal stability condition

$$
\mathcal{E}_{(x, u)}=\left\langle\frac{\partial F_{0}}{\partial x}\right\rangle_{\mathcal{E}_{(x, u)}}+\left\langle\frac{\partial\left(F_{0}\right)}{\partial u}\right\rangle_{\mathcal{E}_{(u)}}+\left\langle\left.\frac{\partial F}{\partial v}\right|_{v=0}, 1\right\rangle_{\mathbb{R}}+\widetilde{F}_{0}^{*} \mathcal{E}_{(\lambda, \mu)},
$$

where $\widetilde{F}_{0}=\left(F_{0}, u\right), F_{0}(x, u)=F(x, u, 0),(\lambda, \mu) \in \mathbb{R} \times \mathbb{R}^{2}$, is weaker than the $R \times L^{+}$-infinitesimal stability condition.

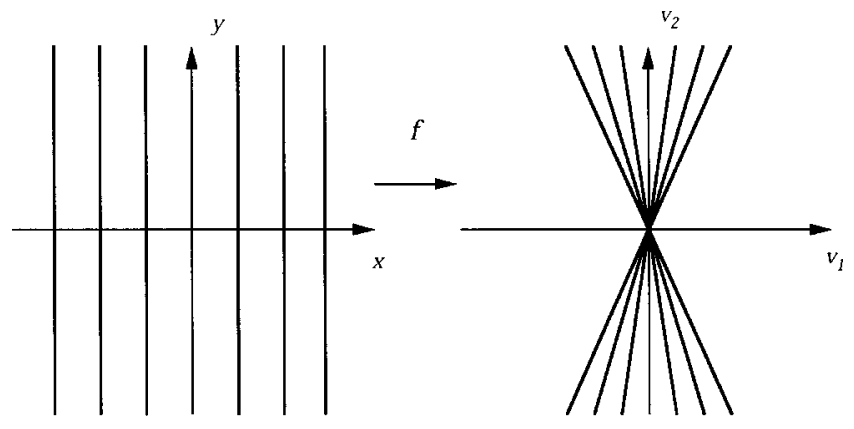

FIG. 4. The vertical lines are mapped onto the lines through the origin by the pinch map. 
If $h(x, u, v)$ is an $(r, s)$-infinitesimally stable unfolding of $f_{0}(x)=F(x, 0,0)$ then the prenormal form for infinitesimally $R \times L^{+}$-stable unfolding of $f_{0}$ is given in the form

$$
F(x, u, v)=h(x, u, v)+\sum_{i=1}^{2} u_{i} g_{i}(u, v),
$$

where $g_{i} \in \mathcal{E}_{(u, v)}$.

By $(r, s)$-stability theory (cf. Ref. 17), if $g \in \mathcal{E}_{(x, u, v)}$ is an $(r, s)$-stable unfolding of $\eta(x)$ $=g(x, 0,0)$, then $g$ is $(r, s)$-equivalent to the function-germ

$$
F\left(x, T_{\sigma}(u, v)\right),
$$

where $F$ is an $(r+s)$-stable unfolding of $\eta$ and $T_{\sigma}(u, v)=T \circ W_{\sigma}$ is a composition of the polynomial mapping $T:\left(\mathbb{R}^{2} \times \mathbb{R}^{2}, 0\right) \rightarrow\left(\mathbb{R}^{2} \times \mathbb{R}^{2}, 0\right)$ and permutation of the variables $W_{\sigma}$. Here we have

$$
T(u, v)=\left(u, v+p(u)+\sum_{i=1}^{s} v_{i} \xi_{i}(u)\right),
$$

where $p(u)$ is a polynomial mapping $\mathbb{R}^{r} \rightarrow \mathbb{R}^{s}$ with zero constant term and degree at most $s+1$ and $\xi(u)$ are polynomial mappings $\mathbb{R}^{r} \rightarrow \mathbb{R}^{s}$ with zero constant terms and degree at most $s-1$. The permutation $W_{\sigma}$ acting on $u, v$-variables, $W_{\sigma}\left(w_{1}, \ldots, w_{r+s}\right)=\left(w_{\sigma(1)}, \ldots, w_{\sigma(r+s)}\right)$ is defined as one of the following permutations; Taking $k \leqslant \min \{r, s\}$ and integers $1 \leqslant i_{1}<\cdots<i_{k} \leqslant r, 1 \leqslant j_{1}<\cdots$ $<j_{k} \leqslant s$ we define $\sigma$ as the product of the following transpositions: $\sigma=\left(i_{1}, r+j_{1}\right)\left(i_{2}, r\right.$ $\left.+j_{2}\right) \cdots\left(i_{k}, r+j_{k}\right)$.

In our (2,2)-case all stable unfoldings are related to the corresponding strata of the family of mappings:

$$
T(u, v)=\left(u_{1}, u_{2}, v_{1}+\sum_{1 \leqslant i+j \leqslant 3} a_{i j} u_{1}^{i} u_{2}^{j}+\sum_{i, j=1}^{2} b_{i j} v_{i} u_{j}, \quad v_{2}+\sum_{1 \leqslant i+j \leqslant 3} c_{i j} u_{1}^{i} u_{2}^{j}+\sum_{i, j=1}^{2} d_{i j} v_{i} u_{j}\right) .
$$

These unfoldings were classified in Refs. 18 and 19 and we may use them in our classification of gravitational caustics.

Remark 6.3: By the straightforward application of the classification theorem from Ref. 18 we find that the generic perturbations of the composed function-germs $F(x, u, v)=F_{1}(x, u)$ $+F_{2}(u, v)$, are $(2,2)$-equivalent to the following normal forms:

$$
\begin{gathered}
F(x, u, v)=x_{1}^{3} \pm x_{2}^{2}+x_{1} u_{1}, \\
F(x, u, v)=x_{1}^{3} \pm x_{2}^{2}+x_{1}\left(v_{1}+u_{1}^{2}+ \pm u_{2}^{2}\right), \\
F(x, u, v)=x_{1}^{3} \pm x_{2}^{2}+x_{1}\left(v_{1}-u_{1}^{2}-u_{2}^{2}\right), \\
F(x, u, v)=x_{1}^{3} \pm x_{2}^{2}+x_{1}\left(v_{1}+u_{1}^{3}+ \pm u_{2}^{2}+u_{1} v_{2}\right), \\
F(x, u, v)= \pm x_{1}^{4} \pm x_{2}^{2}+x_{1}^{2} u_{1}+u_{2} x_{1}, \\
F(x, u, v)= \pm x_{1}^{4} \pm x_{2}^{2}+x_{1}^{2}\left( \pm u_{1}^{2}+u_{2}\right)+x_{1}\left(u_{2}+v_{1}\right), \\
F(x, u, v)= \pm x_{1}^{4} \pm x_{2}^{2}+x_{1}^{2}\left(u_{1}^{3}+u_{1} v_{2}+u_{2}\right)+x_{1}\left(u_{2}+v_{1}\right), \\
F(x, u, v)= \pm x_{1}^{4} \pm x_{2}^{2}+x_{1}^{2}\left( \pm u_{1}^{2}+u_{2} v_{2}+v_{1}\right)+x_{1} u_{2}, \\
F(x, u, v)= \pm x_{1}^{4} \pm x_{2}^{2}+x_{1}^{2} u_{1}+x_{1}\left(u_{1}^{2} \pm u_{2}^{2}+u_{1} v_{1}+v_{2}\right),
\end{gathered}
$$




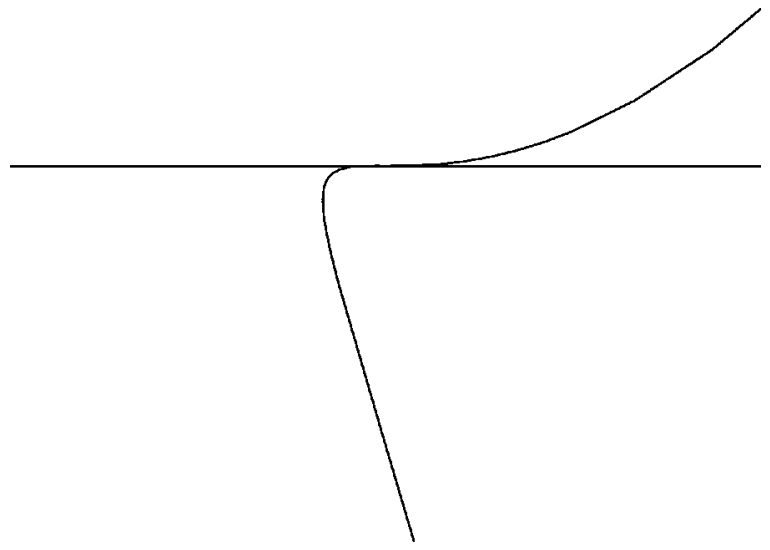

FIG. 5. The picture of the perturbed caustics of the original double lensing system. Twofold curves have order 3 contact at the origin.

$$
\begin{gathered}
F(x, u, v)=x_{1}^{5} \pm x_{2}^{2}+x_{1}^{3} u_{1}+x_{1}^{2}\left(u_{2} v_{2}+c u_{2}+u_{1}+v_{1}\right)+x_{1} u_{2},\left(c \neq-\frac{2}{3}\right), \\
F(x, u, v)=x_{1}^{3}+x_{2}^{3}+x_{1} x_{2} u_{1}+x_{1} u_{2}+x_{2}\left(u_{2} v_{2}+u_{1}+c u_{2}+v_{1}\right),(c \neq 0), \\
F(x, u, v)=x_{1}^{3}-x_{1} x_{2}^{2}+x_{1}^{2}\left(u_{1} v_{2}+c u_{1}+u_{2}+v_{1}\right)+x_{1} u_{2}+x_{2} u_{1},(c \in \mathbb{R}), \\
F(x, u, v)=x_{1}^{3}-x_{1} x_{2}^{2}+x_{1}^{2}\left(u_{2} v_{2}+u_{1}+v_{1}\right)+x_{1} u_{2}+x_{2} u_{1} .
\end{gathered}
$$

By definition, (2,2)-equivalence destroys the exact structure of the composition of caustics. However the structure of generic perturbation of the caustics still remained. By the above list, we can calculate the discriminant set

$$
\mathcal{D}_{F}=\left\{\left(v_{1}, v_{2}\right) \in X_{3} \mid \frac{\partial F}{\partial x_{1}}=\frac{\partial F}{\partial x_{2}}=\frac{\partial F}{\partial u_{1}}=\frac{\partial F}{\partial u_{2}}=0\right\} .
$$

Such sets are the perturbed caustics of the original double lensing systems. For the function germ $F(x, u, v)= \pm x_{1}^{4} \pm x_{2}^{2}+x_{1}^{2} u_{1}+x_{1}\left(u_{1}^{2} \pm u_{2}^{2}+u_{1} v_{1}+v_{2}\right) \quad$ we have $\mathcal{D}_{F}=\{(t, 0)\} \cup\left\{\left(6 s^{2} \pm 2 s\right.\right.$, $\left.\left.\pm 20 s^{3}\right)\right\}$ which is depicted in Fig. 5. We can observe that two regular curves (i.e., folds) have order 3 contact at the origin. These two regular curves are the locus of fold points. Therefore, this is a double fold at the origin.

\section{ACKNOWLEDGMENTS}

S.I. partially supported by Grant-in-Aid for Scientific Research, JSPS, No. 14604003. S.J. partially supported by KBN Grant No. 2 P03A 02017.

${ }^{1}$ A. O. Petters, H. Levine, and J. Wambsganss, Singularity Theory and Gravitational Lensing (Birkhäuser, Boston, 2001). ${ }^{2}$ A. O. Petters and F. J. Wicklin, J. Math. Phys. 39, 1011 (1998).

${ }^{3}$ P. S. Schneider, J. Ehlers, and E. E. Falco, Gravitational Lenses (Springer, Berlin, 1992).

${ }^{4}$ V. I. Arnol'd, S. M. Gusein-Zade, and A. N. Varchenko, Singularities of Differentiable Maps (Birkhäuser, Boston, 1986), Vol. I.

${ }^{5}$ V. M. Zakalyukin, Funct. Anal. Appl. 10, 23 (1976)

${ }^{6}$ A. O. Petters, J. Math. Phys. 34, 3555 (1993).

${ }^{7}$ H. I. Levine, A. O. Petters, and J. Wambsganss, J. Math. Phys. 34, 4781 (1993).

${ }^{8}$ H. I. Levine and A. O. Petters, Astron. Astrophys. 272, L17 (1993).

${ }^{9}$ S. Janeczko, J. Math. Phys. 41, 5642 (2000).

${ }^{10}$ A. O. Petters, J. Math. Phys. 36, 4263 (1995).

${ }^{11}$ S. Janeczko, Math. Proc. Cambridge Philos. Soc. 100, 9 (1986).

${ }^{12}$ S. Janeczko, Ann. Inst. Henri Poincare, Sect. A 56, 429 (1992).

${ }^{13}$ M. Golubitsky and V. Guillemin, Stable Mappings and Their Singularities, GTM 14 (Springer, New York, 1973). 
${ }^{14}$ J. Martinet, Singularities of Smooth Functions and Maps, London Mathematical Society Lecture Note Series Vo. 58 (Cambridge University Press, New York, 1982).

${ }^{15}$ A. Weinstein, Lectures on Symplectic Manifolds, CBMS Regional Conference Series in Mathematics Vol. 29 (AMS, Providence, 1977).

${ }^{16}$ C. G. Gibson, K. Wirthmüller, A. A. du Plessis, and E. J. N. Looijenga, Topological Stability of Smooth Mappings, Lecture Notes in Mathematics Vol. 552 (Springer, New York, 1976).

${ }^{17}$ G. Wassermann, Acta Math. 135, 57 (1975).

${ }^{18}$ X. Jianfei, Acta Math. Sinica, New Series 12, 415 (1996)

${ }^{19}$ I. Nakai, Seminar Notes in Differential Topology, Kyoto University, February 1985. 